\title{
Determining the Gamma-Ray Burst Rate as a Function of Redshift
}

\author{
Nevin Weinberg*, Carlo Graziani ${ }^{\dagger}$, Donald Q. Lamb ${ }^{\dagger}$ and Daniel E. Reichart* \\ ${ }^{*}$ Department of Astronomy, California Institute of Technology, Mail Code 105-24, 1201 East California \\ Boulevard, Pasadena, CA 91125 \\ ${ }^{\dagger}$ Department of Astronomy \& Astrophysics, University of Chicago, 5640 South Ellis Avenue, Chicago, IL 60637
}

\begin{abstract}
We exploit the 14 gamma-ray bursts (GRBs) with known redshifts $z$ and the 7 GRBs for which there are constraints on $z$ to determine the GRB rate $R_{\mathrm{GRB}}(z)$, using a method based on Bayesian inference. We find that, despite the qualitative differences between the observed GRB rate and estimates of the SFR in the universe, current data are consistent with $R_{\mathrm{GRB}}(z)$ being proportional to the SFR.
\end{abstract}

\section{INTRODUCTION}

There is increasing evidence that GRBs are due to the collapse of massive stars (see, e.g., [1] for a discussion of this evidence). If GRBs are indeed related to the collapse of massive stars, one expects the GRB rate to be roughly proportional to the SFR. However, the observed redshift distribution of GRBs differs noticeably from that of the SFR: the observed GRB redshift distribution peaks at $z \approx 1$ and few bursts are observed beyond $z \sim 1.5$, while the SFR peaks at $z \approx 2$ and $10-40 \%$ of stars are thought to form beyond $z=5$ (see, e.g., [2,3,]).

However, observational selection effects play an important role in determining the observed redshift distribution of GRBs. The important question is therefore whether or not the discrepancy between the observed GRB redshift distribution and the redshift dependence of the SFR is entirely due to selection effects; i.e., is the GRB rate roughly proportional to the SFR after taking observational selection effects into account? We address this question in this paper.

\section{METHOD}

We adopt a Bayesian approach. We calculate the likelihood of the data given the model, and convert it to a posterior distribution on the model parameters. We assume a very general model for the GRB rate, and a powerlaw model for the intrinsic GRB photon luminosity distribution (we assume that the amplitude and the powerlaw index of the photon luminosity distribution does not evolve; we relax this assumption in future work). We de- termine the efficiency with which BeppoSAX and the IPN detect GRBs as a function of peak photon flux $P$ by comparing the BeppoSAX and IPN peak photon flux distributions to that of BATSE. We fit the model jointly to the peak fluxes and redshifts of the 14 GRBs with known $z$, and the 7 GRBs for which there are constraints on $z$.

We write the rate of GRBs that occur per unit redshift and luminosity as

$$
d N / d z d L_{N}=\rho(z) f\left(L_{N}\right),
$$

where

$$
\rho(z)=R_{\mathrm{GRB}}(z ; P, Q) \times(1+z)^{-1} \times 4 \pi r(z)^{2}(d r / d z)
$$

is the rate of GRBs that occur at redshift $z$,

$$
R_{\mathrm{GRB}}(z ; P, Q)=\left[\frac{t(z)}{t(0)}\right]^{P} \exp \left[Q\left(1-\frac{t(z)}{t(0)}\right)\right]
$$

is the rate of GRBs that occur at redshift $z$ per unit comoving volume (see [5]), $t(z)$ is the elapsed time since the Big Bang, and

$$
f\left(L_{N}\right)=L_{N}^{-\beta} \times \Theta\left(L_{N}-L_{\min }\right) \Theta\left(L_{\max }-L_{N}\right)
$$

is the intrinsic photon luminosity distribution of GRBs. Thus the model has five parameters: $P, Q, \beta, L_{\min }$, and $L_{\text {max }}$.

We write the efficiency with which GRBs with known redshifts are found as $\varepsilon(z, P)=\varepsilon_{z}(z) \times \varepsilon_{\mathrm{ST}} \varepsilon_{P}(P)$, where $\varepsilon_{z}(z)$ is the efficiency with which the redshifts of GRBs are determined from optical observations once they are detected by a $\gamma$-ray burst instrument. We take $\varepsilon_{z}(z)=1$ 


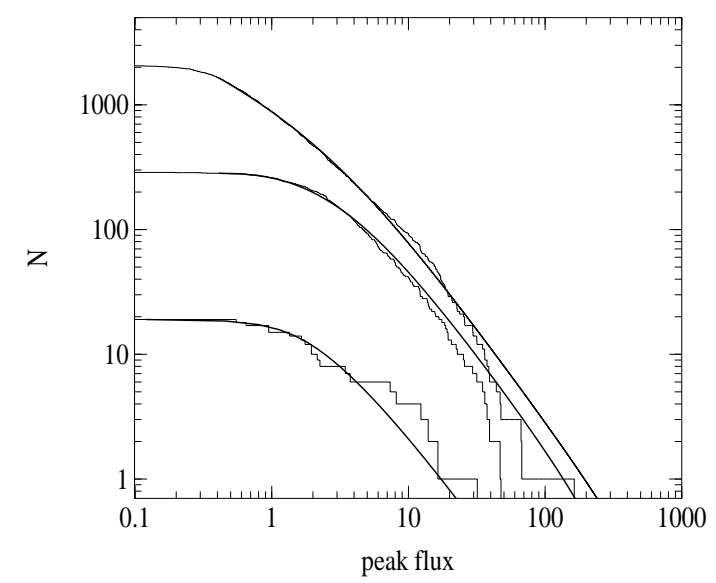

FIGURE 1. Comparison of the best-fit models to the differential distribution of peak fluxes of BATSE, IPN and BeppoSAX bursts, and the cumulative peak flux distributions for the three experiments.

for GRBs whose redshifts were determined by detection of an absorption-line system in the optical afterglow of the burst and $\varepsilon_{z}(z)=\Theta(1-z)+\Theta(z-2.5)$ for GRBs whose redshifts were determined by measuring emission lines in the spectra of the host galaxy. The latter expression accounts qualitatively for the difficulty in measuring redshifts when the $H_{\alpha}$ and $\mathrm{O}[\mathrm{II}]$ emission lines from host galaxies do not lie in the visible spectrum. The quantity $\varepsilon_{\mathrm{ST}}$ is the "stereo-temporal" efficiency that accounts for limitations of exposure in time and solid angle and $\varepsilon_{P}(P)$ is the efficiency with which BATSE, the IPN and BeppoSAX detect GRBs as a function of peak flux $P$.

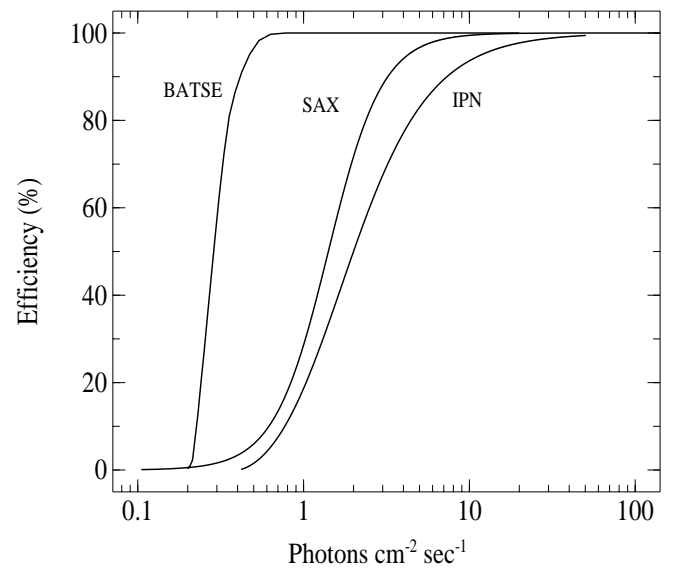

FIGURE 2. Efficiency $\varepsilon(P)$ with which BATSE, IPN and BeppoSAX detect GRBs as given by the best-fit models to the differential distribution of peak fluxes of BATSE, IPN and BeppoSAX bursts.
Figure 1 compare the best-fit models of $\varepsilon_{P}$ and the cumulative peak flux distributions of BATSE, the IPN, and BeppoSAX, respectively. Figure 2 shows the best-fit $\varepsilon_{P}$ for each of the three experiments.

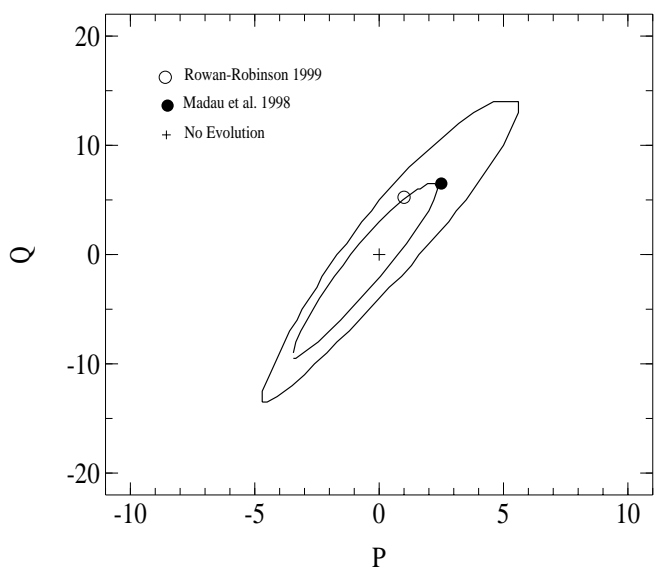

FIGURE 3. Probability contours in the (P,Q)-plane for the GRB rate parameters $(P, Q)$ found from fitting to the 14 GRBs with known $z$ and the 7 GRBs with constraints on $z$. The solid curves correspond to the $68 \%$ and $95 \%$ probability contours. Also shown are the $(\mathrm{P}, \mathrm{Q})$-values corresponding to no space density evolution (+), the Madau et al. SFR [4], and the RowanRobinson phenomenological model fit to IR, optical and UV data [5].

The likelihood function is then given by

$$
\mathcal{L}=\exp \left\{-\int d z d P \mu(z, P) \varepsilon(z, P)\right\} \prod_{i=1}^{N} \mu\left(z_{i}, P_{i}\right),
$$

where

$$
\begin{aligned}
\mu(z, P) & =\int_{0}^{\infty} d L_{N} \rho(z) f\left(L_{N}\right) \delta\left(P-\frac{L_{N}}{4 \pi r(z)^{2}(1+z)^{\alpha}}\right) \\
& =\rho(z) \times f\left(4 \pi r(z)^{2}(1+z)^{\alpha} P\right) \times 4 \pi r(z)^{2}(1+z)^{\alpha}
\end{aligned}
$$

is the expected number of events observed within $d z d P$ of $(z, P)$. The quantity $\alpha$ is the burst spectral index, which we set equal to one in this work. By an application of Bayes' Theorem, we now regard $\mathcal{L}$ as an (unnormalized) probability distribution on the model parameters.

\section{RESULTS}

Figure 3 shows $68 \%$ and $95 \%$ probability contours for the GRB rate parameters $P$ and $Q$. Also shown on the plots are the best-fit SFR models of Madau et al. [4] and Rowan-Robinson [5]. The SFR models lie at about a 68\% excursion from the best-fit GRB rate model. Thus we find that, despite the qualitative differences that exist between the observed GRB rate and estimates of the SFR in the 
universe, current data are consistent with the actual GRB rate being approximately proportional to the SFR.

\section{REFERENCES}

1. Lamb, D. Q. 2000, Phys. Reports, 333, 505

2. Gnedin, N. Y., \& Ostriker, J. P. 1997, ApJ, 486, 581

3. Valageas, P., \& Silk, J. 1999, A\&A, 347, 1

4. Madau, P., Pozzetti, L., \& Dickinson, M. 1998, ApJ, 498, 106

5. Rowan-Robinson, M. 1999, Ap\&SS, 266, 291 